Trauma Berufskrankh 2004 - 6 [Suppl 2] : S266-S272 DOI 10.1007/s10039-003-0775-3

Online publiziert: 26. August 2003

(c) Springer-Verlag 2003

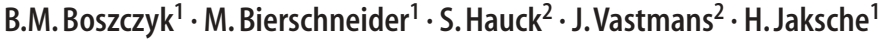

${ }^{1}$ Abteilung für Neurochirurgie, BG-Unfallklinik, Murnau

${ }^{2}$ Abteilung für Unfallchirurgie, BG-Unfallklinik, Murnau

\title{
Kyphoplastie im Vergleich zur Vertebroplastie
}

\author{
Heutiger Stand
}

\section{Entwicklungsgeschichte}

Im Gegensatz zum primären Einsatz der Vertebroplastie bei Hämangiomwirbeln wurde die Technik der Kyphoplastie speziell für die Anwendung am osteoporotischen Knochen entwickelt. Neben der Frakturstabilisierung sollte dieses Verfahren auch eine Frakturreposition ermöglichen. Erreicht wurde diese Kombination durch spezielle Ballons, welche minimalinvasiv in den Wirbelkörper eingebracht werden und eine Frakturreposition durch schrittweise, druckkontrollierte Füllung ermöglichen. Nach Entfernen der Ballons verbleibt ein Hohlraum, welcher mit einem Augmentationsmaterial, in der Regel Polymethylmethacrylat (PMMA), ausgefüllt wird.

Nach Zulassung durch die Amerikanische FDA im Juli 1998 wurde die erste Operation am Patienten im September 1998 in den USA durch Mark Reiley, MD, durchgeführt. Die erste Veröffentlichung zu dieser Methode erschien im Jahr 2000 [29]. Seither wurden nach Angaben des Herstellers (Kyphon - Stand September 2002) in den USA und in Europa bei mehr als 17.000 Patienten über 22.000 Wirbel durch Kyphoplastie augmentiert.

\section{Literaturdaten zur Kyphoplastie}

\section{Klinische Daten}

Im Gegensatz zur Kyphoplastie liegen für die Vertebroplastie bereits veröffentlichte klinische Studien mit einer Nachuntersuchungsdauer von 5-7 Jahren vor [2, 21].

Für beide Verfahren wurde in mehreren Studien eine vergleichbare Schmerzreduktion bei korrekter Indikationsstellung in $80-95 \%$ der Patienten belegt [2, 13, 17, 19]. Die durchschnittliche Reduktion in der visuellen Analogskala lag für die Kyphoplastie bei 4,2-7,8 Punkten, ebenso zeigte sich eine signifikante Verbesserung der Parameter „bodily pain“ und „physical function“ des SF 36 [19].

Bisher liegen zur Kyphoplastie prospektive Untersuchungen an über $200 \mathrm{~Pa}$ tienten mit einem Nachuntersuchungszeitraum bis $\mathrm{zu} 2$ Jahren sowie retrospektive Untersuchungen an über 1400 Patienten mit einem Nachuntersuchungszeitraum bis zu 18 Monaten vor [8].

Eine randomisierte Multicenterstudie, konservativ gegen operativ, wurde im Frühjahr 2003 begonnen.

Eine Differenzierung der klinischen Resultate zwischen osteoporotischen und traumatischen Frakturtypen steht aus. Die erfolgreiche Anwendung bei neoplastischem Befall der Wirbelsäule, insbesondere bei multiplem Myelom, belegten 2 Studien $[11,12]$.

\section{Morphologische Daten}

Eine Zusammenfassung der Ergebnisse aus der Literatur zeigt bezüglich der Frakturreposition eine durchschnittliche Wiederherstellung von $43-47 \%$ des in der
Mittellinie gemessenen Höhenverlusts [2]. Innerhalb der ersten 3 Monate kann es zu einem sekundären Höhenverlust von 3-8\% kommen, darüber hinaus bleibt die Wirbelkörperhöhe nach bisherigen Kenntnissen konstant [8].

Die Korrektur der kyphotischen Fehlstellung eines Wirbels ist deutlich vom Frakturalter abhängig und liegt durchschnittlich bei bis zu $18^{\circ}$, bzw. $50 \%[2,13]$. Bei frischen Frakturen kann eine nahezu vollständige Reposition erreicht werden [6]. Im Schnitt nimmt das Volumen des frakturierten Wirbels um 13,7\% zu [8].

\section{Biomechanische Daten}

Zur biomechanischen Eignung der Kyphoplastie liegen derzeit 3 maßgebliche Untersuchungen vor $[1,27,28]$, in welchen eine Wiederherstellung der Festigkeit des frakturierten Wirbels über das Ausgangsmaß hinaus beobachtet wurde $[1,28]$. Mit Kyphoplastie bzw. mit Vertebroplastie augmentierte Wirbel zeigen sowohl unter maximaler Kompression $[1,28]$ als auch unter zyklischer Dauerbelastung vergleichbare Festigkeiten [27].

Um diese Effekte zu erzielen, ist bei der Kyphoplastietechnik eine komplette Materialfüllung der Kaverne im Wirbelkörper notwendig [27]. Eine spongiöse Verzahnung des Augmentationsmaterials scheint zur Vermeidung von Scherkräften an der Knochen-Material-Grenze Voraussetzung zu sein. 


\section{Operationsindikationen}

Unterschieden wird die Kyphoplastie bei

- osteoporotischen Wirbelkörpersinterungen,

- traumatischen Wirbelfrakturen und

- neoplastischen Wirbelkörpereinbrüchen.

Ausgiebige Erfahrung ist in der perkutanen Anwendung der Kyphoplastie an der Lendenwirbelsäule und der mittleren bis unteren Brustwirbelsäule (BWK5-BWK12) vorhanden [8]. Für die Anwendung an der oberen BWS liegen noch keine Routineerfahrungen vor. Über eine Anwendung an der Halswirbelsäule ist bisher nicht berichtet worden.

In Abhängigkeit vom Höhenverlust, von der Ausdehnung einer Fragmentierung der Wirbelkörperhinterwand und vom Vorliegen neurologischer Ausfälle wird

- die konventionelle perkutane Technik oder

- die mikrochirurgische „halboffene“

Technik

angewendet $[5,6,13]$.

\section{Osteoporotische Frakturen}

Die größte Erfahrung liegt hinsichtlich der Behandlung osteoporotischer Frakturen vor. Indiziert ist das Verfahren bei symptomatischen Frakturen der mittleren Brustwirbelsäule bis Lendenwirbelsäule. Da osteoporotische Wirbelfrakturen ohne neurologische Ausfälle grundsätzlich konservativ behandelt werden können und noch keine randomisierte Studie zugunsten der Operation vorliegt, richtet sich die Operationsindikation nach der Schmerzsymptomatik und der Deformität des Wirbels bzw. der zu erwartenden Progredienz einer Deformität. Im letzteren Fall empfiehlt sich eine frühzeitige Operation, da ein Höhengewinn stark vom Frakturalter abhängig ist.

\section{Neoplastische Wirbelfrakturen}

Im Bereich der neoplastischen Wirbelkörpersinterungen besteht eine Indika-

Trauma Berufskrankh 2004 - 6 [Suppl 2] : S266-S272

DOI 10.1007/s10039-003-0775-3

C Springer-Verlag 2003

B. M. Boszczyk · M. Bierschneider $\cdot$ S. Hauck · J.Vastmans $\cdot$ H. Jaksche

\section{Kyphoplastie im Vergleich zur Vertebroplastie. Heutiger Stand}

\section{Zusammenfassung}

Die Kyphoplastie ist eine innovative, für die minimalinvasive Augmentation osteoporotischer Wirbelfrakturen entwickelte Technik. Sie erlaubt im Gegensatz zur Vertebroplastie eine vom Frakturalter abhängige Reposition durch druckkontrollierte Füllung eines transpedikulär in die Spongiosa des frakturierten Wirbelkörpers eingebrachten Ballonkatheters. Zur Retention wird die nach Ballonentfernung verbleibende Kaverne mit Augmentationsmaterial aufgefüllt. Die dabei erreichte niedrige Rate an ernsten Komplikationen rechtfertigt den Versuch einer Indikationserweiterung auf spezielle traumatische Frakturtypen und neoplastische Wirbeleinbrüche durch osteolytische Metastasen. Neben den konventionellen perkutanen Zugängen ist auch der "halboffene" mikrochirurgische Zugang möglich, der die Anwendung der Kyphoplastietechnik auch bei komplexeren Wirbelkörperfrakturen mit Komprimierung der neuralen Strukturen erlaubt. Kyphoplastie und Vertebroplastie führen aufgrund der sofortigen Stabilisierung des betroffenen Wirbelkörpers über eine schnell einsetzende Schmerzreduktion zur rasch möglichen Mobilisierung der Patienten. Dennoch ist weiterhin ein interdisziplinäres Therapiekonzept gefordert, um neben der operativen Intervention die individuelle medikamentöse und rehabilitative Behandlung der Grunderkrankung zu optimieren.

\section{Schlüsselwörter}

Kyphoplastie · Vertebroplastie - Osteoporose Wirbelfraktur

\section{Comparison of kyphoplasty and vertebroplasty}

\section{Abstract}

Kyphoplasty is a novel method that has been developed for the augmentation of osteoporotic vertebral fractures by minimally invasive techniques. In contrast to vertebroplasty, depending on how old the fracture is, the kyphoplasty technique allows its reduction by controlled inflation of a special balloon introduced through the pedicle into the cancellous bone of the fractured vertebral body, creating a cavity for augmentation. The reduced risk of serious complications, such as epidural leakage of augmentation material, justifies an attempt to extend the indications for this technique to traumatic fractures with involvement of the posterior vertebral wall and to neoplastic vertebral collapse resulting from osteolytic metastases. As well as the conventional percutaneous approaches, the microsurgical interlaminary approach is also possible, allowing the application of kyphoplasty for more complex frac- tures involving compression of the neural structures. The operative kyphoplasty and vertebroplasty techniques bring about swift pain relief and allow rapid mobilisation of patients through the immediate stabilisation of the affected vertebral bodies. Nonetheless, in addition to the operative intervention, medical treatment of the primary disease and rehabilitation treatment of the individual patient should also be optimised, which still demands an interdisciplinary approach.

\section{Keywords}

Kyphoplasty · Vertebroplasty · Osteoporosis . Vertebral fracture 
tion für die Kyphoplastie in erster Linie bei disseminiertem Tumorbefall durch vorwiegend osteolytische Metastasen [11, 12]. Die Operationstechnik unterscheidet sich grundsätzlich nicht von der Anwendung bei osteoporotischen oder traumatischen Frakturen.

Je nach Ausprägung der Osteolyse ergeben sich Einschränkungen für die Kyphoplastietechnik, so :

- bei weitgehender Destruktion der Kortikalis, welche zu einem Durchbruch des Ballons führen kann,

- bei ausgedehntem Verlust der trabekulären Spongiosastruktur, wodurch eine spongiöse Verzahnung des Augmentationsmaterials verhindert wird.

Im Einzelfall müssen daher die isolierte Versorgung durch Kyphoplastie oder das zusätzliche Einbringen einer internen Fixierung abgewogen werden.

Vorteilhaft ist das geringe Operationstrauma der perkutanen Technik, welche eine weitere Behandlung durch Chemotherapie oder Bestrahlung nur um wenige Tage verzögert.

Theoretisch besteht ein Risiko der venösen Zellaussaat durch die Verdrängung der Tumormasse im Wirbelkörper. Obwohl wissenschaftliche Belege für eine solche Aussaat derzeit nicht vorliegen, empfiehlt sich die Kyphoplastie in erster Linie bei disseminiertem Tumorbefall ohne kurative operative Sanierungsmöglichkeit im Bereich der Wirbelsäule.

\section{Operationstechniken mit Falldiskussion}

Unterschieden werden

- die konventionellen perkutanen Techniken über den trans- und extrapedikulären Zugang und

- die „halboffene“ mikrochirurgische interlaminare Technik $[5,6,13]$.

Die perkutanen Verfahren sind sowohl in Allgemeinnarkose als auch in örtlicher Betäubung durchführbar. In Vollnarkose muss besonders auf eine vorsichtige Lagerung und Polsterung der osteoporotischen Patienten in Bauchlage geachtet werden, da es durch unvorsichtige Lagerungsmaßnahmen leicht zu Rippenfrakturen kommen kann.

Die intraoperative Bildgebung ist mit Bildwandlertechnik möglich (zitiert nach [7]).Als Zeit sparend hat sich die Anwendung von 2 Bildwandlern, für die simultane Bildgebung in 2 Ebenen, erwiesen. Auch bei Durchführung der Kyphoplastie in der CT ist ein Bildwandler empfehlenswert, um bei der Augmentation des Wirbelkörpers durch eine engmaschige Durchleuchtung einen paravertebralen oder epiduralen Zementaustritt sofort erkennen zu können.

\section{Perkutane transpedikuläre Kyphoplastie}

Alle Operationsschritte erfolgen in zeitlicher Abfolge beidseitig. Die Haut wird mit einer Stichinzision kraniolateral des Pedikeleintrittspunkts an der kraniolateralen Pedikelzirkumferenz eröffnet. Die Stärke des Weichteilmantels muss für die Konvergenz der Stichrichtung berücksichtigt werden. Eine Knochenbiopsienadel wird auf den Übergang zwischen Querfortsatz und kranialem Gelenkfortsatz aufgesetzt (Position 1, • Abb. 1a,b). Mit leichten Hammerschlägen wird die Biopsienadel in den Pedikel eingetrieben und im seitlichen Strahlengang bis zur Pedikeltaille vorgetrieben (Position 2,• Abb. 1a). Sie darf dabei im a.-p.-Strahlengang die mediale Pedikelkortikalis nicht überqueren (Position 2, - Abb. 1b). Bei korrekter Lage wird die Nadel weiter bis zur Pedikelbasis an der Wirbelkörperhinterwand vorangetrieben (Position 3 , - Abb. 1a) und darf sich erst jetzt im a.-p.Strahlengang auf die mediale Pedikelkortikalis projizieren (Position 3, $\bullet$ Abb. 1b). Durch strenges Einhalten dieser radiomorphologischen Landmarken wird eine spinale Perforation vermieden. Die Nadel wird anschließend bis in das anteriore Wirbelkörperdrittel eingetrieben (Position 4, • Abb. 1a,b). Über die Biopsienadel wird ein Kirschner-Draht eingebracht (Schritt A, • Abb. 1a), welcher als Führung für den kanülierten Arbeitstrokar dient, der über den Draht in den Wirbelkörper eingetrieben wird (Schritt B, • Abb. 1a). Nach Entfernen des Kirschner-Drahts und des Arbeitstrokars verbleibt die Arbeits- kanüle im posterioren Wirbelkörperdrittel.

Bei sämtlichen anschließenden Arbeitsschritten im Wirbelkörper muss eine Perforation der Kortikalis sorgfältig vermieden werden, da bei der Injektion des Augmentationsmaterials jede Perforationsstelle einen Punkt des geringsten Widerstands darstellt, über welchen es zu einem Materialaustritt kommen kann. Über die Arbeitskanüle kann eine Biopsie des Wirbelkörpers entnommen werden. Anschließend wird mit einem Handbohrer eine zur Mittellinie konvergierende Bohrung bis in das vordere Wirbelkörperdrittel gefertigt (Schritt C, • Abb. 1a). In diese Bohrung wird der ungefüllte Kyphoplastieballon platziert (Schritt D, - Abb. 1a). Die Ballongröße wird in Abhängigkeit von der Wirbelkörpergröße gewählt (15 mm Länge mit 4 ml Füllvolumen oder $20 \mathrm{~mm}$ Länge mit $6 \mathrm{ml}$ Füllvolumen). Die endgültige Lage der nun beidseitig eingebrachten Ballons wird in beiden Ebenen mit Bildwandler verifiziert.

Über ein manuelles Druckinjektionssystem kann ein Druck bis etwa $2800 \mathrm{kPa}$ ( 400 PSI) über den kontrastmittelgefüllten Ballon auf die angrenzenden Strukturen ausgeübt werden. In der Regel genügt jedoch bei korrekter Platzierung ein Druck von etwa $700 \mathrm{kPa}$ ( 100 PSI). Durch die schrittweise, druckkontrollierte Füllung der Ballons werden die verletzte Spongiosa komprimiert und die angrenzende Endplatte gehoben ( $\bullet$ Abb. 1c). Dies erfolgt unter Durchleuchtung in 2 Ebenen, wobei auf eine laterale, ventrale oder posteriore Perforation der Kortikalis geachtet werden muss. Nach erreichter Frakturreposition bei frischen Frakturen oder nach Erzeugung einer ausreichenden Kaverne bei älteren osteoporotischen Frakturen werden die Kyphoplastieballons geleert und entfernt.

Die verbleibende Kaverne (Defektzone) im Wirbelkörper wird mit Augmentationsmaterial (in der Regel PMMA) über eine Applikationskanüle aufgefüllt (- Abb. 1d). Um einen epiduralen oder paravertebralen Materialaustritt zu vermeiden, sollte das Augmentationsmaterial mit hoher Viskosität und niedrigem Druck eingefüllt werden. Das Füllvolumen ist von dem zuletzt erreichten Volumen der Kyphoplastieballons bekannt und 
wird geringfügig überschritten, um eine spongiöse Verzahnung zu erreichen (- Abb. 1e).

\section{Perkutane extrapedikuläre Kyphoplastie}

Der perkutane Zugang an der BWS kann transpedikulär in der gleichen Technik wie an der LWS erfolgen. Im Bereich der mittleren BWS ist jedoch der Pedikelverlauf in der Regel stärker an der Sagittalebene orientiert, sodass bei den meist schmalen Pedikeln keine ausreichende Konvergenz in das anteriore Wirbelkörperdrittel erreicht werden kann.

Eine stärkere Konvergenz bietet der so genannte extrapedikuläre Zugang. Hierbei wird die Knochenbiospienadel kranial des Querfortsatzes mit konvergierender und abfallender Stichrichtung in die Rinne zwischen Rippenhals und lateraler Pedikelkortikalis eingebracht. Dadurch kommt es zu einer stärkeren Konvergenz und einer Perforation der lateralen Pedikelkortikalis nahe der Pedikelbasis. Das Einbringen des Kyphoplastieballons und die Augmentation werden in gleicher Weise vorgenommen, wie für den transpedikulären Zugang beschrieben. Bei kleineren Wirbeln der mittleren BWS kann ein einzelner, konvergent eingebrachter Ballon genügen.

\section{Fallberichte}

\section{Fall 1}

Der 69-jährige Patient erlitt bei einem Sturz eine Keilfraktur von LWK1, Typ A1.2, die zunächst konservativ versorgt wurde. Aufgrund persistenter, erheblicher Rückenschmerzen nach mehrwöchiger konservativer Therapie wünschte der Patient eine operative Versorgung. In der MRT (- Abb. 2a) wurden ein Frakturödem und eine leichte kyphotische Fehlstellung des gebrochenen LWK1 gesehen. Kyphoplastieballons wurden perkutan in die Frakturzone eingebracht ( $\bullet$ Abb. 2b), die frakturierte Spongiosa wurde durch die druckkontrollierte Füllung der Ballons komprimiert und somit die Deckplatte gehoben (• Abb. 2c). Nach Entfernen der Ballons verblieb im Bereich der Fraktur im Wirbelkörper eine Kaverne (Defekt-

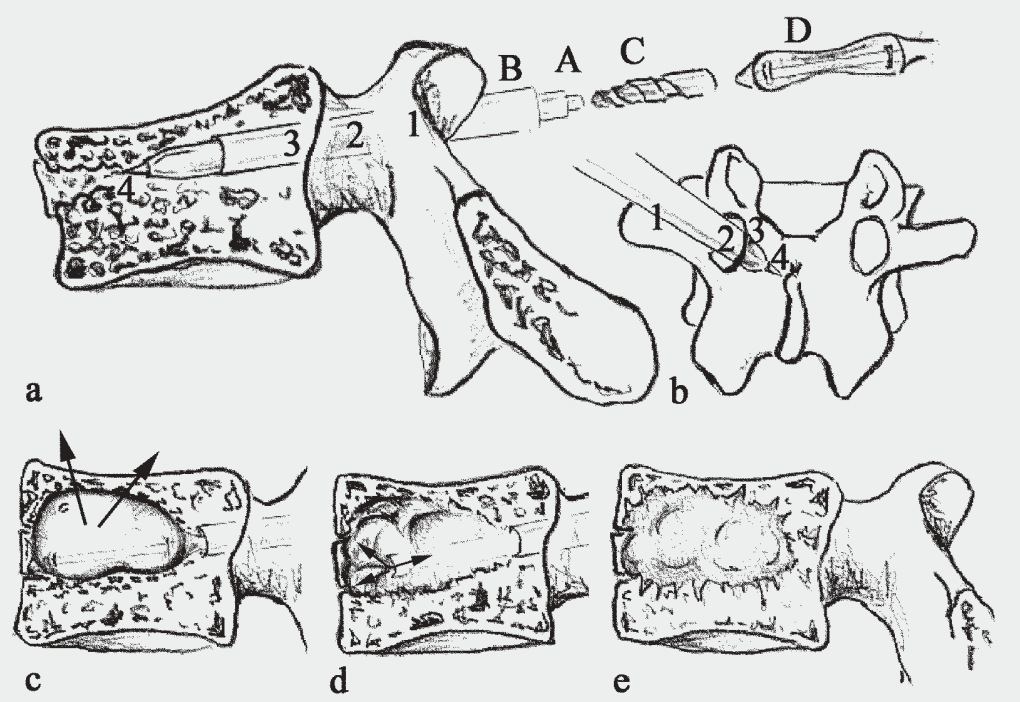

Abb. 1a-e $\Delta$ Schematische transpedikuläre Kyphoplastie, Lendenwirbelkörper mit Keilfraktur, Typ A1.2, alle Operationsschritte beidseitig, a,b transpedikuläre Platzierung eines Kyphoplastieballons, Ansicht von a lateral, b dorsal; 1 Ansetzen der Biopsienadel am Pedikeleintrittspunkt im Winkel zwischen oberem Gelenkfortsatz und Querfortsatz, 2 Passage der Pedikeltaille ohne Perforation der medialen Pedikelkortikalis, 3 Eintritt in den Wirbelkörper, 4 endgültige Lage der Nadel im medialen, anterioren Drittel des Wirbelkörpers, $A$ Kirschner-Draht durch die Biopsienadel als Führungsdraht, $B$ Einbringen des kanülierten Arbeitstrokars über Führungsdraht, $C$ Einbringen des Handbohrers durch die Arbeitskanüle in die Frakturzone D Platzierung des Kyphoplastieballons in den Bohrkanal in der Frakturzone, c druckkontrollierte Füllung des Kyphoplastieballons und Aufrichtung des Wirbelkörpers, d Auffüllen der Kaverne mit hochviskösem Augmentationsmaterial über die Applikationskanüle, e mit der Spongiosa verzahnter Augmentationsbereich in der ehemaligen Frakturzone, s. auch Text

zone) (• Abb. 2d), die mit hochviskösem PMMA aufgefüllt wurde (• Abb. 2e).

Das postoperative Röntgenbild zeigte eine Korrektur der leichten kyphotischen Fehlstellung und eine Verteilung des PMMA unter der Deckplatte und in der Spongiosa des Wirbelkörpers (• Abb. 2f). Die postoperative CT $(\bullet \mathrm{Abb} .2 \mathrm{~g})$ belegte die Auffüllung des Frakturbereichs unterhalb der kranialen Deckplatte ohne PMMA-Austritt.

Die Operation dauerte $30 \mathrm{~min}$, der Blutverlust war $<20 \mathrm{ml}$. Bereits am ersten postoperativen Tag konnte der Patient nahezu beschwerdefrei voll mobilisiert werden.

\section{Fall 2}

Die 77-jährige Patientin litt nach einem häuslichen Sturz an ausgeprägten Rückenschmerzen. Im CT zeigte sich eine inkomplette Berstungsfraktur (Typ A3.1) von LWK1 bei vorbestehender Osteoporose (- Abb. 3a,b). Beide angrenzenden Wirbel und die gesamte untere Lenden- wirbelsäule wiesen osteoporotische Frakturen auf. Eine Abgrenzung weiterer frischer Frakturen mit MRT war aufgrund einer Platzangst der Patientin nicht möglich. Beim Vorliegen multipler Frakturen wurde die Indikation zur perkutanen, transpedikulären Kyphoplastie mit PMMA von BWK12-LWK5 gestellt. Bei LWK1 erfolgte die Perforation der Pedikel nahe der Basis, um eine Manipulation der vermuteten Pedikelfraktur zu minimieren.

Die Operationszeit betrug $150 \mathrm{~min}$ bei einem Blutverlust von $<50 \mathrm{ml}$. In der postoperativen CT bestand kein Hinweis für epiduralen oder paravertebralen Materialaustritt (• Abb. 3c,d).

Bei deutlicher Schmerzreduktion war die Patientin am ersten postoperativen Tag mit Rollator mobilisierbar.

\section{Komplikationen}

Insgesamt ist die in der Literatur angegebene Komplikationsrate sehr niedrig. In einer Zusammenfassung der ersten 2194 

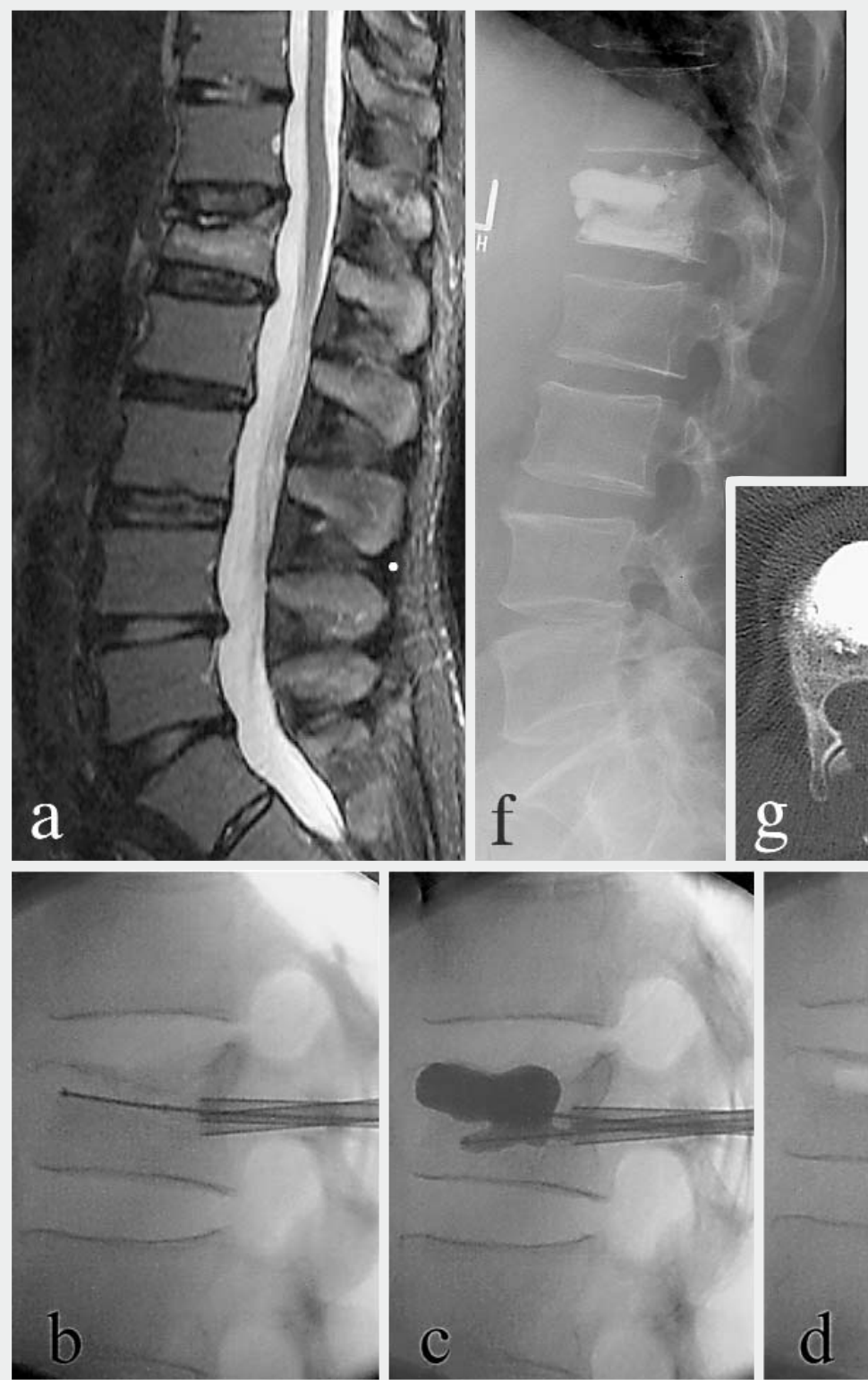

Abb. 2a-g $<$ Perkutane transpedikuläre Kyphoplastie, Keilfraktur des ersten Lendenwirbelkörpers, 69-jähriger Patient, a sagittale MRT der LWS, b in die Frakturzone eingebrachter KyphoplastiebalIon, c druckkontrollierte Füllung der Kyphoplastieballons, d verbleibende Kaverne (Defektzone) nach Entfernen der Kyphoplastieballons, e mit PMMA augmentierter Wirbelkörper, f postoperatives seitliches Röntgenbild, g postoperatives axiales $\mathrm{CT}$ des augmentierten ersten Lendenwirbelkörpers
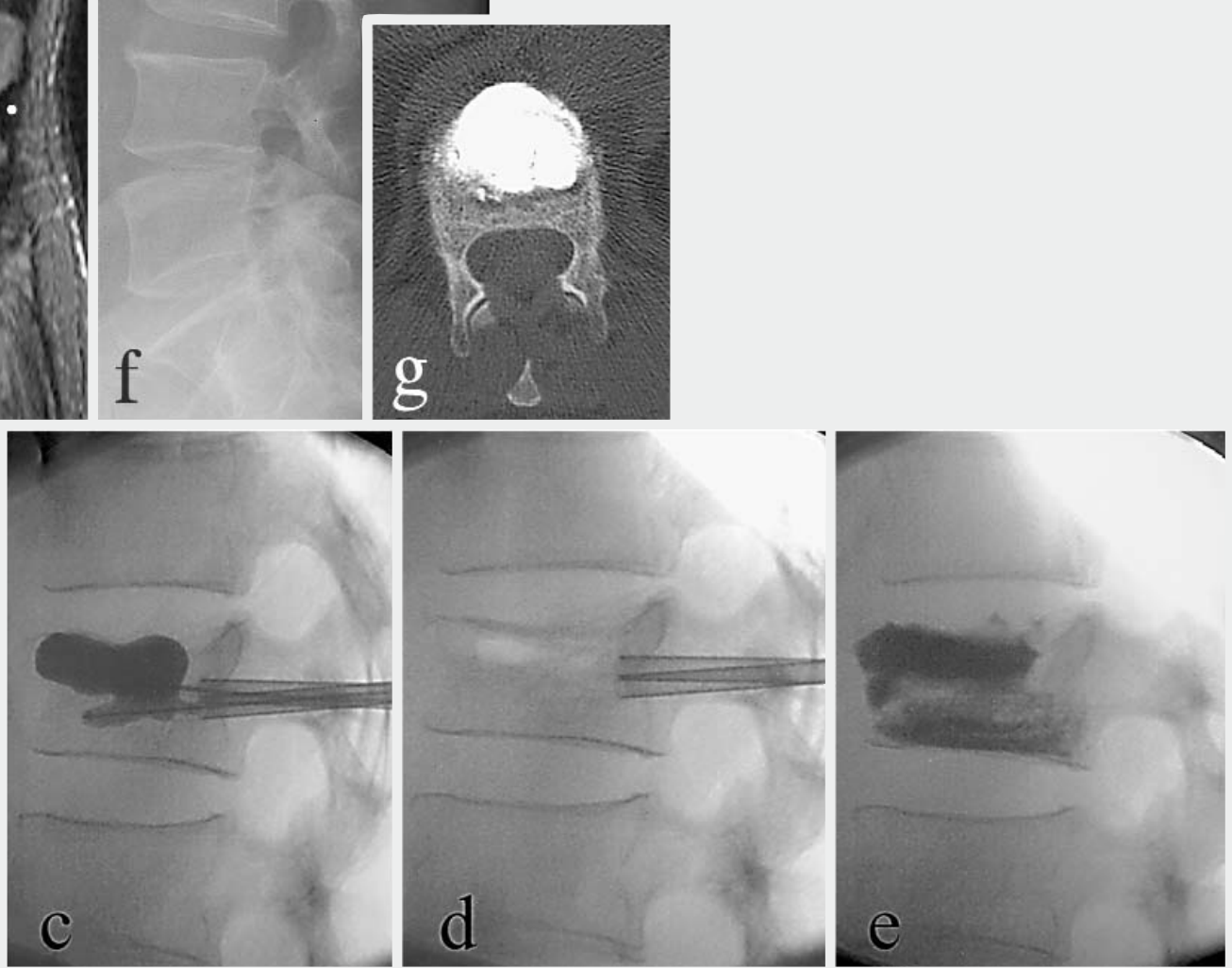

Operationen ergab sich eine Rate an ernsthaften Komplikationen von $0,2 \%$ pro Fraktur [14]. Zugangsbedingt wurden in den ersten 100 Fällen 3 thorakale Paraparesen durch spinale Instrumentenfehllage, ein A.-spinalis-anterior-Syndrom durch beidseitige infrapedikuläre Fehllage und ein spinales epidurales Hämatom bei systemischer Antikoagulation gesehen. Ähnliche Komplikationen sind seither nicht veröffentlicht worden [8].

\section{Austritt von PMMA}

Die häufigste Komplikation stellt der Austritt von PMMA dar. Im Vergleich zur Ver- tebroplastie fällt diese Rate jedoch deutlich geringer aus [8]. PMMA-induzierte Lungenembolien, paradoxe zerebrale Embolien und thorakale Paraparesen, wie sie für die Vertebroplastie in Einzelfällen beschrieben worden sind $[4,9,15,16,18,20$, 22, 24, 25], fanden sich für die Kyphoplastie nicht. Dennoch sollte für den Fall einer Komplikation eine operative Interventionsmöglichkeit gegeben sein.

\section{Fettembolie}

Eine bisher wenig untersuchte Komplikationsmöglichkeit stellt die Fettembolie dar. Beim Aufdehnen der Ballons im Be- reich des Wirbelkörpers wird Knochenmark verdrängt, welches über den venösen Abfluss in die Lungenstrombahn gelangt. Entsprechende Veränderungen und Reaktionen der Lungenstrombahn, wie sie z. B. aus der Hüftendoprothetik bekannt sind, sind anzunehmen. Eine suffiziente Überwachungsmöglichkeit für diese Problematik besteht bisher nicht.

\section{Risiko einer angrenzenden Fraktur}

Das Risiko einer angrenzenden Fraktur nach Augmentation wird kontrovers diskutiert. Hier lässt sich noch keine endgültige Entscheidung fällen, ob eine an- 
grenzende Fraktur grundsätzlich als Komplikation zu werten ist. Biomechanisch ergab sich kein Beleg für eine erhöhte Steifigkeit der Wirbel im normalen Belastungsbereich $[1,28]$,jedoch wird die Frakturschwelle angrenzender Wirbel herabgesetzt [3]. Klinisch wurde in der größten retrospektiven Studie von 2194 Frakturen allerdings keine erhöhte Inzidenz von angrenzenden Frakturen in der mittelfristigen Nachuntersuchung gefunden [14]. Insgesamt scheint beim Vorhandensein einer einzelnen, nicht spontanen Fraktur ein sehr geringes Risiko (in einzelnen Berichten $<3 \%$ ) einer neuen angrenzenden Spontanfraktur zu bestehen [8].

Bei Patienten mit multiplen Spontanfrakturen ist jedoch das Risiko für eine neu auftretende Wirbelfraktur auch ohne Operation deutlich erhöht (bei 2 Frakturen bis 7 fach, bei mehr als 3 Frakturen bis über 17fach) [23]. Da insbesondere im thorakolumbalen Übergang die Inzidenz neuer Frakturen erhöht ist [10], kann bei Augmentation in diesem Bereich leicht der Eindruck einer operationsbedingt erhöhten Frakturrate erweckt werden. Bei Patienten mit multiplen Spontanfrakturen erscheint daher eine Ausweitung der Augmentation auf angrenzende Wirbel des thorakolumbalen Übergangs sinnvoll. Wissenschaftliche Untersuchungen hierzu liegen allerdings noch nicht vor.

\section{Schlussfolgerung}

Für Patienten mit osteoporotischen und disseminierten neoplastischen Wirbeleinbrüchen stellen die konventionellen, implantatgebundenen Verfahren aufgrund ihrer Invasivität oftmals keine praktikable Therapieform dar. Die Technik der Kyphoplastie und Vertebroplastie, als minimalinvasive Verfahren, haben bei sorgfältiger Indikationsstellung innerhalb dieser Krankheitsbilder bereits sehr gute erste klinische Ergebnisse gezeigt. Die sofortige Stabilität der behandelten Wirbelkörper erzielt eine schnell einsetzende Schmerzreduktion und erlaubt eine rasche Mobilisierung der Patienten. Dabei kann meist auf eine Korsettversorgung verzichtet werden. Die klinischen Ergebnisse der Kyphoplastie sind mit denen der Vertebroplastie vergleichbar, zeichnen sich jedoch durch die deutlich geringere Rate
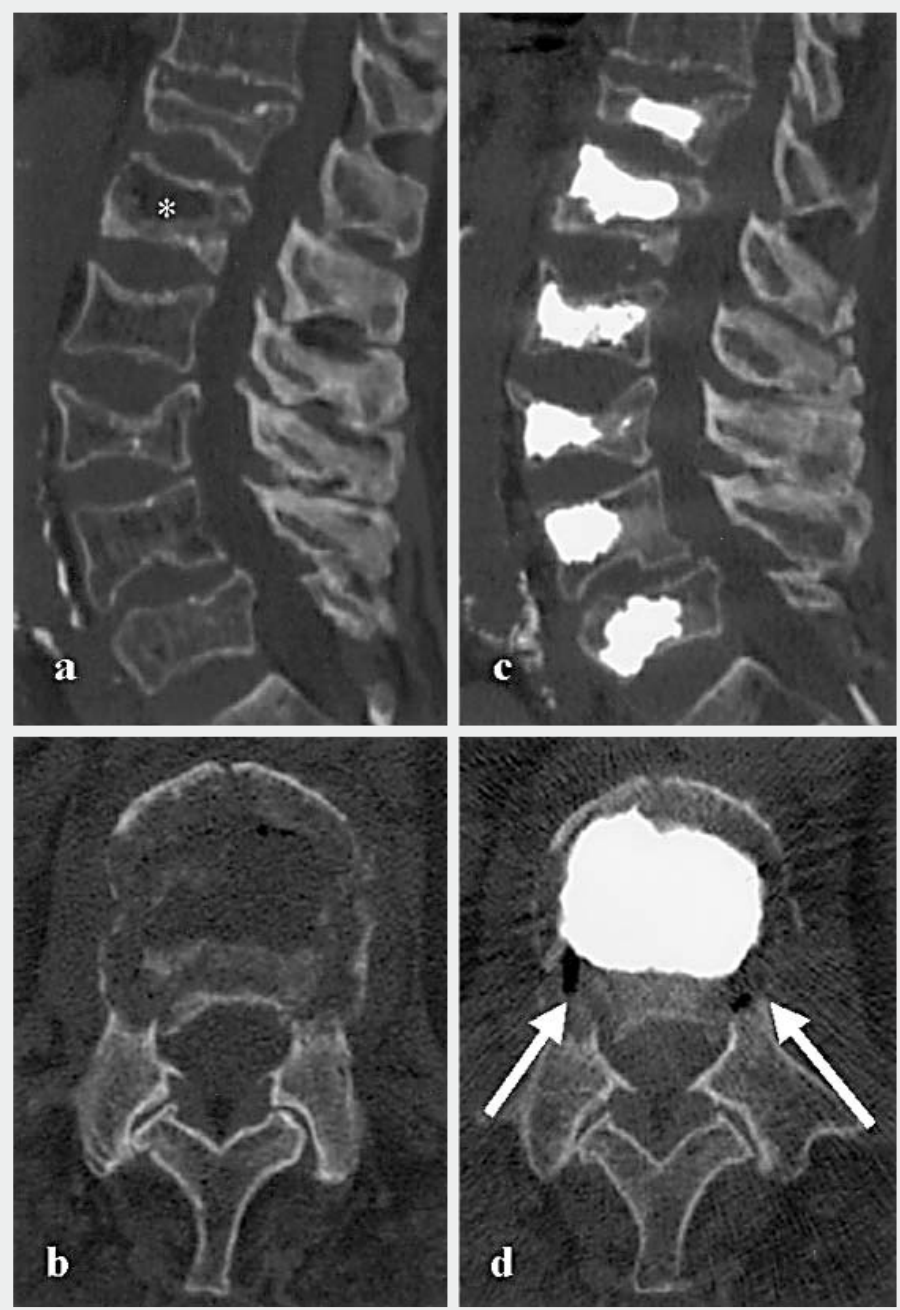

Abb. 3a-d $\Delta$ Perkutane transpedikuläre Kyphoplastie, 77-jährige Patientin mit multiplen osteoporotischen Frakturen und inkompletter Berstungsfraktur (Typ A3.1) von LWK1; a sagittale CT-Rekonstruktion der LWS, Nachweis von Frakturen von BWK12-LWK5, Stern LWK1; b axiale CT von LWK1, Nachweis einer Hinterkantenfraktur mit beidseitiger Ausstrahlung in die Pedikelbasis; $c, d$ postoperative sagittale (c) und axiale (d) CT, Nachweis der PMMA-Lage innerhalb der Wirbelkörper nach perkutaner Kyphoplastie von BWK12-LWK5, bei LWK1 Perforation der Pedikel nahe der Basis (weiße Pfeile)

an ernsthaften Komplikationen aus. Das Hauptrisiko eines unkontrollierten Austritts von Augmentationsmaterial in den Epiduralraum oder in die Lungenstrombahn mit dem Risiko der Embolisierung wird durch die Augmentation mit geringem Druck und hoher Materialviskosität in eine präformierte Kaverne im Wirbelkörper sichtlich gemindert. Damit besteht für die Kyphoplastie eine Erweiterungsmöglichkeit auf die Behandlung von komplexeren Frakturen mit Fragmentierung oder Osteolysen der Hinterwand. In Abhängigkeit vom Ausmaß der Verletzung der Hinterwand und dem Vorhandensein einer neuralen Kompressionssymptomatik kann die Wahl zwischen der konventionellen perkutanen und der halboffenen mikrochirurgisch interlaminaren Technik getroffen werden.

Abschließend muss betont werden, dass die beschriebenen Operationstechniken, insbesondere bei osteoporotischen und neoplastischen Wirbelfrakturen, nicht das Ziel haben, die Therapie der Grunderkrankung zu ersetzen. Hier ist weiterhin ein interdisziplinäres Therapiekonzept gefordert, um die individuelle operative, medikamentöse und rehabilitative Behandlung zu optimieren. 


\section{Korrespondierender Autor \\ Dr. B.M. Boszczyk}

Abteilung für Neurochirurgie, BG-Unfallklinik, Prof.-Küntscher-Straße 8, 82418 Murnau,

E-Mail: B.Boszczyk@gmx.net

Danksagung Besonderer Dank gilt Frau Dr. med. Alexandra Boszczyk für die Anfertigung der Zeichnungen.

\section{Literatur}

1. Belkoff SM, Mathis JM, Fenton DC, Scribner RM, Reiley ME, Talmadge K (2001) An ex vivo biomechanical evaluation of an inflatable bone tamp used in the treatment of compression fracture. Spine 26: 151-156

2. Berlemann U, Heini PF (2002) Perkutane Zementierungstechniken zur Behandlung osteoporotischer Wirbelkörpersinterungen. Unfallchirurg 105: 2-8

3. Berlemann U, Ferguson SJ, Nolte LP, Heini PF (2002) Adjacent vertebral failure after vertebroplasty. J Bone Joint Surg Br 84: 748-752

4. Bernhard J, Heini PF, Villiger PM (2003) Asymptomatic diffuse pulmonary embolism caused by acrylic cement: an unusual complication of percutaneous vertebroplasty. Ann Rheum Dis 62: 85-86

5. Boszczyk BM, Bierschneider M, Robert B, Jaksche H (2003) Treatment of severe osteoporotic fractures through a microsurgical interlaminary approach. In: Szpalski M, Gunzburg R (eds) Vertebral osteoporotic compression fractures. Lippincott, Philadelphia, pp 179-188

6. Boszczyk B, Bierschneider M, Potulski M, Robert B, Vastmans J, Jaksche H (2002) Erweitertes Anwendungsspektrum der Kyphoplastie zur Stabilisierung der osteoporotischen Wirbelfraktur. Unfallchirurg 105: 952-957

7. Boszczyk BM, Bierschneider M, Robert B, Jaksche $H$ (2002) Augmentationstechniken an der Wirbelsäule Aktueller Stand der Techniken und der therapeutischen Möglichkeiten. Orthop Rheuma 1: 19-26

8. Boszczyk BM, Bierschneider M, Schmid K, Robert B, Jaksche $H$ (2002) Kypholasty - a review of current knowledge and operative techniques (abstract). Eur Spine J 11: 612

9. Chen HL, Wong CS, Ho ST, Chang FL, Hsu CH, Wu CT (2002) A lethal pulmonary embolism during percutaneous vertebroplasty. Anesth Analg 95: 1060-1062

10. Davis JW, Grove JS, Wasnich RD, Ross PD (1999) Spatial relationships between prevalent and incident spine fractures. Bone 24: 261-264

11. Dudeney S, Lieberman IH, Reinhardt MK, Hussein M (2002) Kyphoplasty in the treatment of osteolytic vertebral compression fractures as a result of multiple myeloma.J Clin Oncol 20: 2382-2387

12. Fourney DR, Schomer DF, Nader R, Chlan-Fourney J, Suki D, Ahrar K, Rhines LD, Gokaslan ZL (2003) Percutaneous vertebroplasty and kyphoplasty for painful vertebral body fractures in cancer patients. J Neurosurg 98: 2130

13. Garfin SR, Hansen AY, Reiley MA (2001) Kyphoplasty and vertebroplasty for the treatment of painful osteoporotic compression fractures. Spine 26: 1511-1515

14. Garfin SR, Lin G, Lieberman I, Phillips F, Truumees E (2001) Retrospective analysis of the outcomes of balloon kyphoplasty to treat vertebral body compression fracture refractory to medical management (abstract). Eur Spine J 10: S7-S8
15. Harrington KD (2001) Major neurological complications following percutaneous vertebroplasty with polymethylmethacrylate. J Bone Joint Surg Am 84: 1070-1073

16. Jang JS, Lee SH, Jung SK (2002) Pulmonary embolism of polymethylmethacrylate after percutaneous vertebroplasty. Spine 27: E416-E418

17. Ledlie JT, Renfro M (2003) Balloon kyphoplasty: oneyear outcomes in vertebral body height restoration, chronic pain and activity levels. J Neurosurg 98: 36-42

18. Lee BJ, Lee SR, Yoo TY (2002) Paraplegia as a complication of percutaneous vertebroplasty with polymethylmethacrylate. Spine 27: E419-E422

19. Lieberman IH, Dudeney S, Reinhardt MK, Bell G (2001) Initial outcome and efficacy of „kyphoplasty ${ }^{\prime \prime}$ in the treatment of painful osteoporotic vertebral compression fractures. Spine 26: 1631-1638

20. Padovani B, Kasriel O, Brunner P, Peretti-Viton P (1999) Pulmonary embolism caused by acrylic cement: $a$ rare complication of percutaneous vertebroplasty. Am J Neuroradiol 20: 375-377

21. Perez-Higueras A, Alvarez L, Rossi RE, Quinones D, Al-Assir I (2002) Percutaneous vertebroplasty: longterm clinical and radiological outcome. Neuroradiology 44: 950-954

22. Ratliff J, Nguyen T, Heiss J (2001) Root and spinal cord compression from methylmethacrylate vertebroplasty. Spine 26: E300-E302

23. Ross PD, Genant HK, Davis JW, Miller PD, Wasnich RD (1993) Predicting vertebral fracture incidence from prevalent fractures and bone density among noneblack, osteoporotic women. Osteoporosis Int 3: 120126

24. Scroop R, Eskridge J, Britz GW (2002) Paradoxical cerebral embolization of cement during intraoperative vertebroplasty: case report. Am J Neuroradiol 23: 868-870

25. Tozzi P, Abdelmoumene Y, Corno AF, Gersbach PA, Hoogewoud HM, Segesser LK von (2002) Management of pulmonary embolism during acrylic vertebroplasty. Ann Thorac Surg 74: 1706-1708

26. Wenger W, Markwalder TM (1999) Surgically controlled, transpedicular methyl methacrylate vertebroplasty with fluoroscopic guidance. Acta Neurochir (Wien) 141: 625-631

27. Wilke HJ, Mehnert U, Boszczyk B, Bierschneider M, Jaksche $H$, Claes LE (2002) Biomechanical evaluation of vertebro- and kyphoplasty under cyclic loading (abstract). Eur Spine J 11: 613

28. Wilson DR, Myers ER, Mathis JM, Scribner RM, Conta JA, Reiley MA, Talmadge KD, Hayes WC (2000) Effect of augmentation on the mechanics of vertebral wedge fractures. Spine 25: 158-165

29. Wong W, Reiley M, Garfin S (2000) Vertebroplasty/Kyphoplasty. JWomens Imaging 2: 117-124 